\title{
Improvement in asthma endpoints when aiming for total control: salmeterol/fluticasone propionate versus fluticasone propionate alone
}

\author{
*Ashley A Woodcock', Alfredas Bagdonas ${ }^{b}$, Watchara Boonsawat ${ }^{c}$, Michael R Gibbs \\ a North West Lung Centre, Wythenshawe Hospital, Manchester, UK \\ ${ }^{\mathrm{b}}$ Red Cross Clinical Hospital, Kaunas, Lithuania \\ Department of Medicine, Faculty of Medicine, Khon Kaen University, Thailand \\ ${ }^{d}$ GlaxoSmithKline R\&D, Greenford, UK \\ e Hôpital Arnaud de Villeneuve, Montpellier, France \\ ${ }^{\ddagger}$ Division of Pulmonology, Department of Medicine, University of Cape Town, Cape Town, South Africa
} Jean Bousquet ${ }^{\mathrm{e}}$, Eric D Bateman', on behalf of the GOAL Steering Committee \& Investigators

Received 13th July 2006; accepted 19th March 2007

\begin{abstract}
Aims: To investigate the magnitude of change in morning peak expiratory flow (PEF), asthma symptoms, and rescue $\beta_{2}$-agonist use, when the aim of treatment is to achieve guideline-defined control.

Methods: This was a protocol-defined analysis of data from the previously-reported one-year, stratified, randomised, double-blind, parallel-group GOAL study comparing the use of salmeterol/fluticasone propionate with fluticasone propionate alone in achieving guideline-defined control; this analysis assessed the magnitude of change in single specific endpoints which were amalgamated into the composite measure of control used in the primary GOAL analysis.

Results: Across all strata, improvements were seen for each outcome at 52 weeks as compared to baseline: mean morning PEF, 58.2 $\mathrm{I} / \mathrm{min}$ (salmeterol/fluticasone propionate) versus $33.9 \mathrm{l} / \mathrm{min}$ (fluticasone propionate alone); symptom scores, -1.0 versus -0.8; symptomfree days, $72.5 \%$ versus $54.5 \%$; mean of zero night awakenings, $31 \%$ versus $22 \%$; rescue-free days, 87.3 versus 74.7 ; annualised rate of severe exacerbations, 0.02 versus $0.03 ; p<0.001$ for all treatment differences.

Conclusions: Aiming for guideline-defined control resulted in sustained, clinically relevant improvements in a range of individual asthma outcomes. Improvements were greatest with salmeterol/fluticasone propionate versus fluticasone propionate alone.

(C) 2007 General Practice Airways Group. All rights reserved.

AA Woodcock, et al. Prim Care Resp J 2007; 16(3): 155-161.

doi:10.3132/pcrj.2007.00043
\end{abstract}

Keywords asthma, control, salmeterol, fluticasone propionate, outcomes

\section{Introduction}

The Global Asthma Insights and Reality (AIR) surveys showed that levels of asthma control worldwide fall far short of the goals set out by the Global Initiative for Asthma (GINA). ${ }^{1,2}$ The one-year Gaining Optimal Asthma controL (GOAL) study investigated the benefits of aiming for comprehensive, guideline-defined control of asthma in patients with suboptimal control. In the GOAL study, guideline-defined asthma control was assessed over eight-week periods using two composite measures derived from the stringent criteria specified in the GINA and National Institutes of Health guidelines. ${ }^{1,3}$ 'Total Control' was defined as none of the following for at least seven out of eight weeks: daytime symptoms; use of rescue medication; night-time awakenings; exacerbations; emergency visits; or treatment-related adverse events enforcing a change in therapy. In addition, patients had to have a morning peak expiratory flow (PEF) of $\geq 80 \%$ predicted for every day of the eight weeks. For 'Well

\footnotetext{
* Corresponding author: Professor Ashley A Woodcock, North West Lung Centre, Wythenshawe Hospital and University of Manchester, Southmoor Road, Manchester M23 9LT, UK. Tel: +44 (0)161 2915873 Fax: +44 (0)161 2915730 Email: ashley.woodcock@manchester.ac.uk
} 
Controlled' asthma, patients had to meet the same criteria as Total Control for night-time awakenings, exacerbations, emergency visits, and treatment-related adverse events. Patients also had to achieve two out of the following each week: $\leq 2$ days with a daytime symptom score $>1$; $\leq 2$ days and $\leq 4$ occasions of rescue medication use; morning PEF $\geq 80 \%$ predicted every day.

As previously reported, the results of the GOAL study showed that comprehensive, guideline-defined control can be achieved and maintained in a population that included patients with a wide range of asthma severities. Guidelinedefined control was achieved by significantly more patients with salmeterol/fluticasone propionate compared with fluticasone propionate alone, more rapidly, and at a lower dose of inhaled corticosteroid (ICS). ${ }^{4}$

The GOAL study used a composite measure of control comprising a number of outcomes commonly used as single endpoints in clinical trials of asthma. In the original GOAL paper, these composite endpoints of Total Control and Well Controlled asthma were reported; however, this obscures the relative magnitude of change in the single endpoints, which would increase our understanding of the benefits of a therapeutic strategy aiming to control asthma completely. Therefore, in this analysis we report the magnitude of change in single specific endpoints - morning PEF, asthma symptoms, symptom-free days, night-time awakenings, rescue $\beta 2$-agonist use, and severe exacerbations - in the GOAL study population.

In addition, patients in the initial study analysis ${ }^{4}$ were stratified according to prior treatment. Here, we also examine the outcomes in a pooled analysis of all strata, which provides an approximation of what might be seen across a range of patients in primary care.

\section{Methods Study design}

The details of the trial design and methods of the GOAL study have been described previously. ${ }^{4}$ Patients were randomised to either salmeterol/fluticasone propionate or fluticasone propionate alone and stratified according to ICS use during the six months before screening: Stratum 1, no ICS; Stratum 2, $\leq 500$ mcg beclometasone dipropionate daily or equivalent; or Stratum 3, >500-1000 mcg beclometasone dipropionate daily or equivalent.

In Phase I of the study, treatments were stepped-up every 12 weeks aiming for Total Control. In Strata 1 and 2, there were up to three treatment steps: salmeterol/fluticasone propionate 50/100,50/250,50/500 mcg twice daily or fluticasone propionate 100, 250, 500 mcg twice daily. In Stratum 3, the dose could be increased in two steps: salmeterol/fluticasone propionate 50/250,50/500 mcg twice daily or fluticasone propionate 250,500 mcg twice daily.
During Phase II, patients remained on the dose at which they had achieved Total Control, or the maximum study dose, until the end of the 52-week treatment period.

\section{Assessment of asthma outcomes}

In the primary analysis, ${ }^{4}$ the primary endpoint was the proportion of patients who achieved Well Controlled asthma during Phase I. The current protocol-defined analysis describes the impact of salmeterol/fluticasone propionate compared with fluticasone propionate alone on the magnitude of change in the individual outcomes that comprised the guideline-defined control criteria in GOAL.

Patients used a daily record card to record the following: morning PEF; asthma symptom score; required use of the short-acting $\beta_{2}$-agonist (SABA) salbutamol during the previous 24 hours; and night-time awakenings.

Peak expiratory flow was measured by patients every morning on waking using a PEF meter (Mini-Wright PEF meter with the ATS volumetric scale [60-880 $\mathrm{mL}$ ] manufactured by Clement Clark, UK).

Patients were asked to record their asthma symptoms over the previous 24 hours before measuring morning PEF and before taking either study medication or SABA. Symptoms recorded included wheeze, shortness of breath, cough, or chest tightness, rated using a $0-5$ scale, where 0 represents no symptoms during the day and 5 represents symptoms of sufficient severity to prevent the patient working or performing normal daily activities.

A severe exacerbation was defined as deterioration in asthma symptoms requiring administration of oral corticosteroids and/or requiring an emergency room visit and/or admission to hospital.

\section{Statistical analyses}

Mean morning PEF and asthma symptoms were analysed using an analysis of covariance with covariates of country amalgamation, age, sex, and baseline. The frequency distribution of rescue medication use and night-time awakenings were analysed using the Van Elteren extension to the Wilcoxon rank sum test ${ }^{5}$ stratified by country groupings. The number of exacerbations was analysed using a maximum likelihood-based analysis assuming the Poisson distribution with covariates adjusted for in the model. To aid clinical interpretation of these data, post hoc assessments of the frequency distribution of percentage of symptom-free days (defined as a 24-hour period with a symptom score of 0 ) and rescue-free days (defined as a 24-hour period without SABA use for relief) were analysed using a proportional odds logistic regression, adjusted for sex, age and country amalgamation. All treatment differences were presented with $95 \%$ confidence intervals. For pooled analyses, strata were included as a covariate in the models; treatment by strata interaction was tested at the $10 \%$ level, and will be mentioned only where significant. 
Table 1. Baseline characteristics in each stratum and in all strata combined.

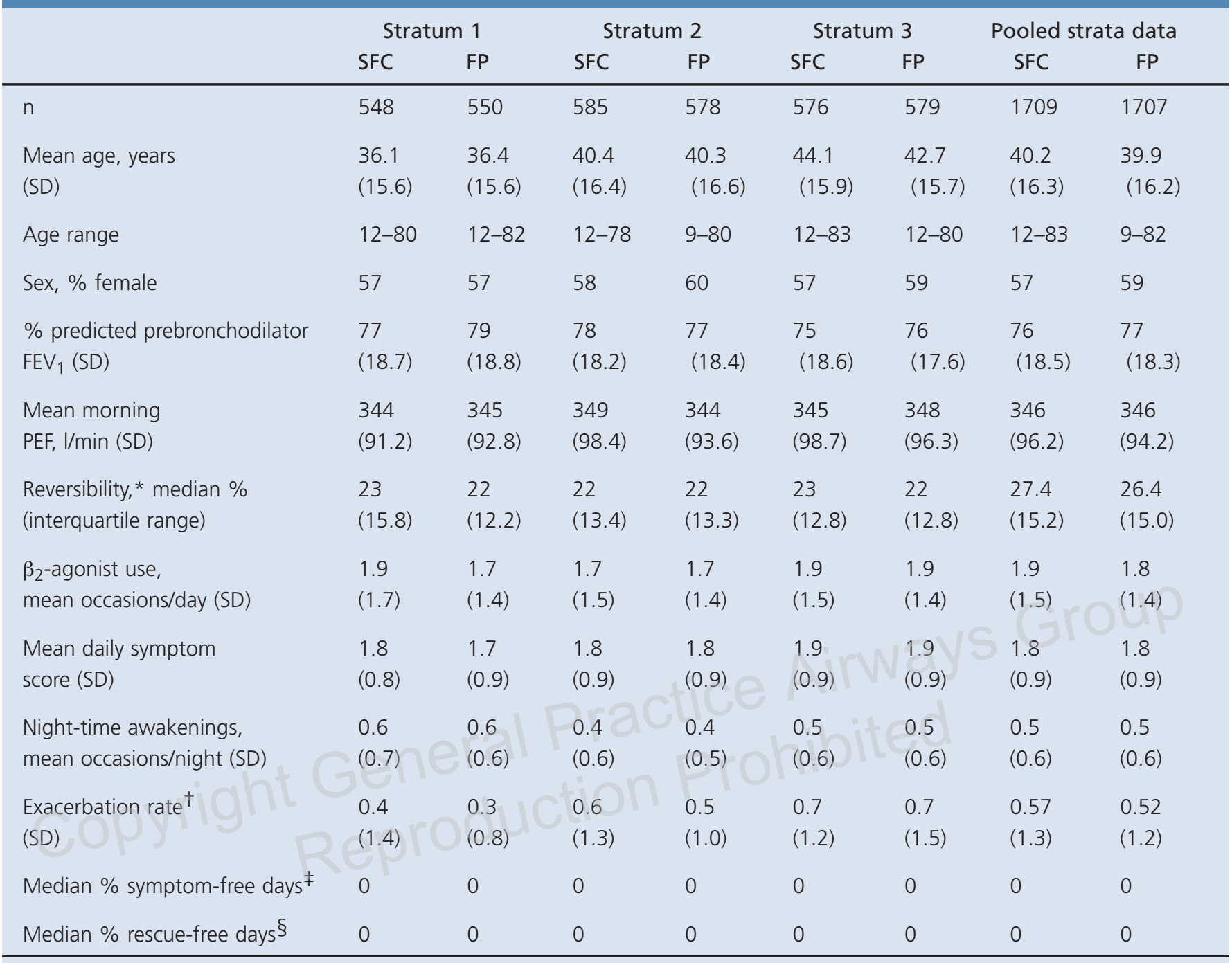

*Patients in whom reversibility was measured during run-in; ${ }^{\dagger}$ documented episodes of hospitalisation and/or course of oral steroids or antibiotics for the treatment of an exacerbation of asthma during the past 12 months; ${ }^{\ddagger}$ post hoc analysis of percentage of symptom-free days; $\S_{\text {post }}$ hoc analysis of percentage of days with no rescue $\beta_{2}$-agonist use

$\mathrm{FEV}_{1}$, forced expiratory volume in 1 second; FP, fluticasone propionate; PEF, peak expiratory flow; SD, standard deviation; SFC, salmeterol/fluticasone propionate

\section{Results}

\section{Baseline characteristics}

Patient numbers and asthma characteristics in the intent-totreat population $(n=3416)$ were comparable between treatment groups within each stratum (see Table 1). A total of 3039 patients completed Phase I and 2890 patients completed Phase II. ${ }^{4}$

\section{Peak expiratory flow}

Mean morning PEF improved substantially on both treatments over the 52-week period across all strata (see Figure 1B). The greatest improvements in PEF were seen in Stratum 1. In all strata, change from baseline in morning PEF was greatest during Weeks 1-12, but continued to improve to Week 52 (see Figure 1).

Overall, there was almost twice as much improvement in morning PEF with salmeterol/fluticasone propionate versus the improvement with fluticasone propionate alone. Over Weeks 1-52, and across all strata, salmeterol/fluticasone propionate improved mean morning PEF by $58.2 \mathrm{l} / \mathrm{min}$ and fluticasone propionate alone improved mean morning PEF by $33.9 \mathrm{l} / \mathrm{min}$ (treatment difference $24.3 \mathrm{l} / \mathrm{min}, \mathrm{p}<0.001$ ).

\section{Asthma symptoms}

Asthma symptom scores improved substantially in all strata for both treatments. At baseline the mean symptom score in all 
A

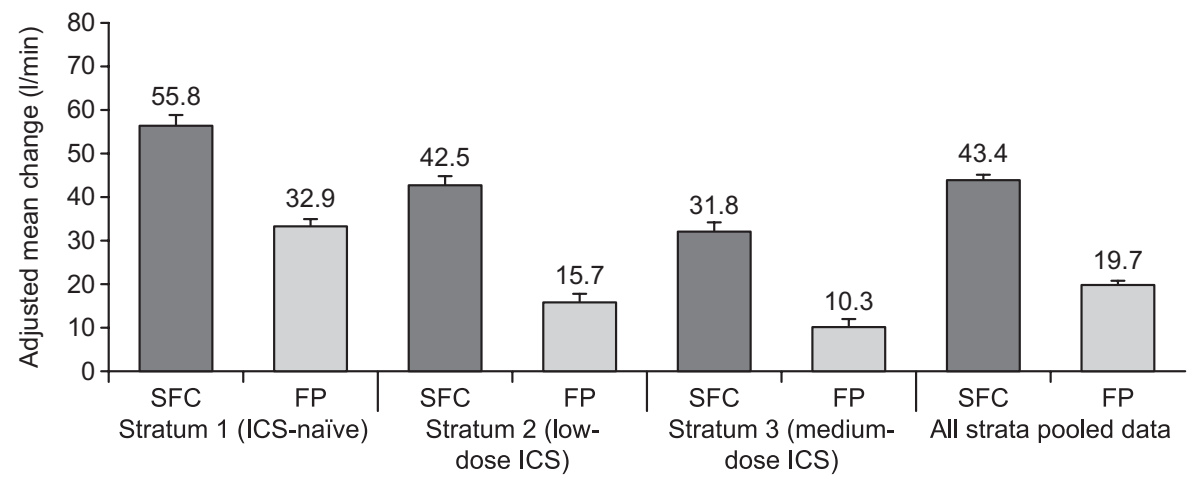

B

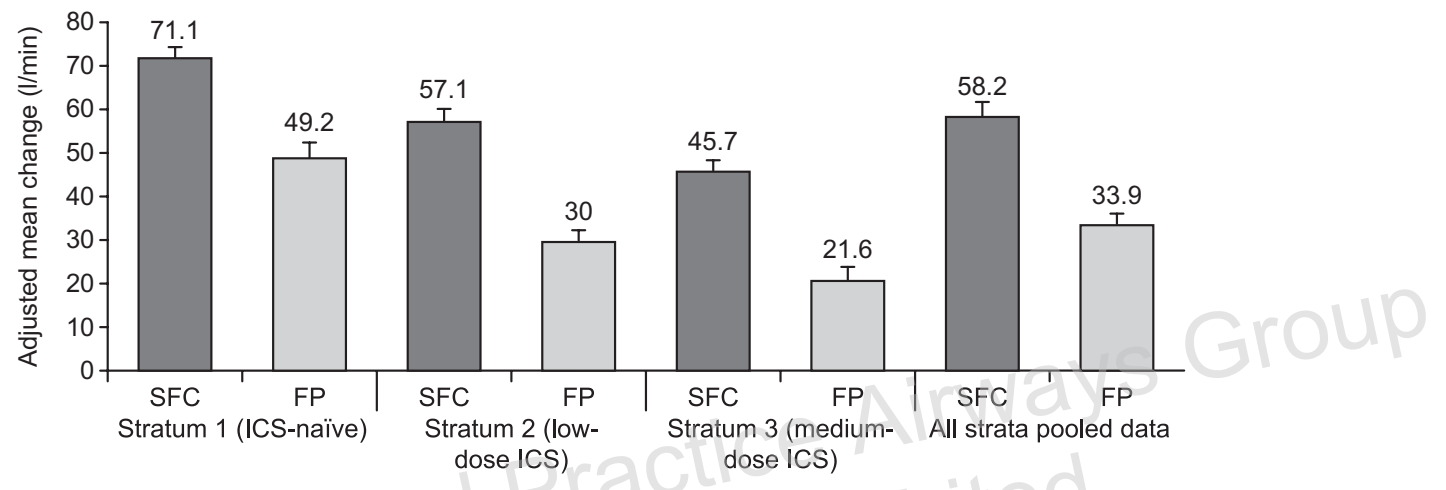

FP, fluticasone propionate; SFC, salmeterol/fluticasone propionate

COOY

strata and in all treatment groups was similar (1.7-1.9). During Weeks $1-52$, salmeterol/fluticasone propionate reduced mean daily symptom scores significantly more than fluticasone propionate alone in each stratum and in the pooled analysis ([p<0.001]; see Table 2).

Over Weeks 1-12 and Weeks 1-52 across all strata, there was greater prevention of symptoms in patients treated with salmeterol/fluticasone propionate versus fluticasone propionate alone, as demonstrated by the significant difference in percentage of symptom-free days (see Table 2). There was a significant treatment by strata interaction (Weeks 1-52, $\mathrm{p}=0.022$ ); no other significant interactions were seen. The pooled analysis showed that patients treated with salmeterol/fluticasone propionate were symptom-free for a median $72.5 \%$ of the time compared with $54.5 \%$ of the study period for patients treated with fluticasone propionate alone. This difference represents an additional 66 symptom-free days a year with salmeterol/fluticasone propionate versus fluticasone propionate alone (18 additional days per year in Stratum 1, 85 in Stratum 2 and 95 in Stratum 3).

\section{Rescue medication use}

In all time periods and all strata, median use of salbutamol in the salmeterol/fluticasone propionate group was significantly lower than in the fluticasone propionate alone group $(p<0.001)$. Over the 52-week study period in Stratum 1, 86\% of patients treated with salmeterol/fluticasone propionate, versus $77 \%$ of patients treated with fluticasone propionate alone, had a median rescue medication use of 0 . The corresponding data for Stratum 2 and 3 were $81 \%$ versus $66 \%$ (Stratum 2), and $71 \%$ versus $57 \%$ (Stratum 3). In the pooled strata analysis, $79 \%$ versus $67 \%$ of patients, had a median rescue medication use of 0 .

Over Weeks 1-12 and Weeks 1-52, the percentage of rescue medication-free days was significantly greater in the salmeterol/fluticasone propionate treatment group versus patients treated with fluticasone propionate alone (all comparisons, $\mathrm{p}<0.001$ ); see Table 3.

\section{Night-time awakenings}

The mean number of awakenings per night was significantly lower $(p<0.05)$ in patients treated with salmeterol/fluticasone propionate versus fluticasone propionate alone during Weeks 1-12 and Weeks 1-52 in each stratum and in the pooled strata analysis. Over Weeks 1-12 in Stratum 1, the proportion of patients with a median of 0 night-time awakenings was 
Table 2. Adjusted mean change in asthma symptom score and median percentage of symptom-free days during Weeks 1-12 and Weeks 1-52.

\begin{tabular}{|c|c|c|c|c|c|c|c|c|}
\hline & \multicolumn{2}{|c|}{$\begin{array}{l}\text { Stratum } 1 \\
\text { (ICS naïve) }\end{array}$} & \multicolumn{2}{|c|}{$\begin{array}{c}\text { Stratum } 2 \\
\text { (low-dose ICS) }\end{array}$} & \multicolumn{2}{|c|}{$\begin{array}{c}\text { Stratum } 3 \\
\text { (medium-dose ICS) }\end{array}$} & \multicolumn{2}{|c|}{ Pooled strata data } \\
\hline & SFC & FP & SFC & FP & SFC & FP & SFC & FP \\
\hline $\begin{array}{l}\text { Weeks } 1-12 \text { adjusted } \\
\text { mean change (SE) }\end{array}$ & $\begin{array}{l}-0.9^{*} \\
(0.0)\end{array}$ & $\begin{array}{l}-0.7 \\
(0.0)\end{array}$ & $\begin{array}{l}-0.8^{*} \\
(0.0)\end{array}$ & $\begin{array}{l}-0.5 \\
(0.0)\end{array}$ & $\begin{array}{l}-0.6^{*} \\
(0.0)\end{array}$ & $\begin{array}{l}-0.3 \\
(0.0)\end{array}$ & $\begin{array}{l}-0.8^{*} \\
(0.0)\end{array}$ & $\begin{array}{l}-0.5 \\
(0.0)\end{array}$ \\
\hline $\begin{array}{l}\text { Weeks } 1-12 \text { median } \% \\
\text { symptom-free days }\end{array}$ & 64.8 & 50.6 & 45.6 & 10.9 & 19.0 & 3.6 & 44.2 & 16.2 \\
\hline $\begin{array}{l}\text { Odds ratio for symptom-free days } \\
\text { during Weeks } 1-12^{\S}(95 \% \mathrm{Cl})\end{array}$ & \multicolumn{2}{|c|}{$1.37^{\dagger}(1.10,1.71)$} & \multicolumn{2}{|c|}{$1.79 *(1.44,2.24)$} & \multicolumn{2}{|c|}{$1.76 *(1.39,2.22)$} & \multicolumn{2}{|c|}{$1.62 *(1.43,1.85)$} \\
\hline $\begin{array}{l}\text { Weeks } 1-52 \text { adjusted } \\
\text { mean change (SE) }\end{array}$ & $\begin{array}{l}-1.2^{*} \\
(0.0)\end{array}$ & $\begin{array}{l}-1.0 \\
(0.0)\end{array}$ & $\begin{array}{l}-1.1^{*} \\
(0.0)\end{array}$ & $\begin{array}{l}-0.8 \\
(0.0)\end{array}$ & $\begin{array}{l}-0.9 * \\
(0.0)\end{array}$ & $\begin{array}{l}-0.6 \\
(0.0)\end{array}$ & $\begin{array}{l}-1.0^{*} \\
(0.0)\end{array}$ & $\begin{array}{l}-0.8 \\
(0.0)\end{array}$ \\
\hline $\begin{array}{l}\text { Weeks } 1-52 \text { median } \% \\
\text { symptom-free days }\end{array}$ & 81.5 & 76.6 & 74.2 & 51.0 & 55.9 & 29.9 & 72.5 & 54.5 \\
\hline $\begin{array}{l}\text { Odds ratio for symptom-free days } \\
\text { during Weeks } 1-52 \S(95 \% \mathrm{Cl})\end{array}$ & \multicolumn{2}{|c|}{$1.30^{\ddagger}(1.03,1.64)$} & \multicolumn{2}{|c|}{$2.06 *(1.66,2.56)$} & \multicolumn{2}{|c|}{$1.78 *(1.43,2.21)$} & \multicolumn{2}{|c|}{$1.69 *(1.49,1.92)$} \\
\hline
\end{tabular}

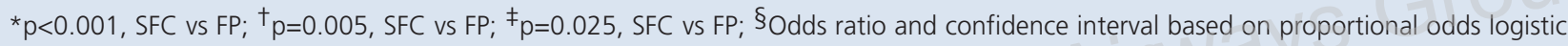
regression analysis

FP, fluticasone propionate; SFC, salmeterol/fluticasone propionate

Table 3. Rescue-free days during Weeks 1-12 and Weeks 1-52.

\begin{tabular}{|c|c|c|c|c|c|c|c|c|}
\hline \multirow[t]{2}{*}{ UUP } & \multicolumn{2}{|c|}{ Stratum 1} & \multicolumn{2}{|c|}{ Stratum 2} & \multicolumn{2}{|c|}{ Stratum 3} & \multicolumn{2}{|c|}{ Pooled strata data } \\
\hline & SFC & FP & SFC & FP & SFC & FP & SFC & FP \\
\hline \multicolumn{6}{|l|}{ Weeks $1-12$ median $\%$} & 33.3 & 75.0 & 53.6 \\
\hline Odds ratio§ $(95 \% \mathrm{Cl})$ & \multicolumn{2}{|c|}{$1.73(1.38,2.19)$} & \multicolumn{2}{|c|}{$2.20(1.77,2.74)$} & \multicolumn{2}{|c|}{$1.84(1.48,2.28)$} & \multicolumn{2}{|c|}{$1.92(1.69,2.18)$} \\
\hline $\begin{array}{l}\text { Weeks } 1-52 \text { median } \% \\
\text { rescue-free days }\end{array}$ & 91.8 & 87.1 & 87.8 & 72.0 & 77.9 & 61.9 & 87.3 & 74.7 \\
\hline Odds ratio§ $(95 \% \mathrm{Cl})$ & \multicolumn{2}{|c|}{$1.64(1.27,2.12)$} & \multicolumn{2}{|c|}{$2.24(1.78,2.82)$} & \multicolumn{2}{|c|}{$1.85(1.48,2.30)$} & \multicolumn{2}{|c|}{$1.91(1.67,2.18)$} \\
\hline
\end{tabular}

$43 \%$ for patients treated with salmeterol/fluticasone propionate versus 35\% for patients treated with fluticasone propionate alone. Corresponding data for Stratum 2, Stratum 3 and the pooled analysis were: $42 \%$ versus $31 \%$ in Stratum 2; $34 \%$ versus $27 \%$ in Stratum 3; and $40 \%$ versus $31 \%$ for the pooled analysis.

Over Weeks 1-52 in Stratum 1, the proportion of patients with a median of 0 night-time awakenings was 33\% for patients treated with salmeterol/fluticasone propionate versus
$28 \%$ for patients treated with fluticasone propionate alone. Corresponding data for Stratum 2, Stratum 3 and the pooled analysis were: $33 \%$ versus $23 \%$ in Stratum 2; $27 \%$ versus $17 \%$ in Stratum 3; and $31 \%$ versus $22 \%$ for the pooled analysis.

\section{Exacerbations}

The mean annual rates of exacerbations requiring oral corticosteroids and/or hospitalisation or emergency visits were significantly lower in the salmeterol/fluticasone propionate group in each stratum $(p<0.009)$ and have been reported 
previously according to phase. ${ }^{4}$ The annualised rate of severe exacerbations requiring hospitalisation or emergency visits was low in both treatment groups across all strata (Stratum 1, 0.02 versus 0.03 visits per year for salmeterol/fluticasone propionate versus fluticasone propionate respectively $[\mathrm{p}=0.014]$; Stratum 2 , 0.02 versus $0.02[p=0.993]$; Stratum $3,0.03$ versus 0.04 $[p=0.007]$; pooled analysis, 0.02 versus $0.03[p<0.001]$ ).

\section{Discussion}

The GOAL study demonstrated that comprehensive, guidelinedefined asthma control is achievable in a wide range of patients. The analysis presented here shows that, over 52 weeks, aiming for Total Control of asthma by stepping up treatment and then sustaining that level of treatment, resulted in patients in both treatment arms achieving substantial benefits in individual outcomes; these include mean morning PEF, asthma symptoms, night-time awakenings, the need for rescue medication, and severe exacerbations.

These findings are in line with the results of the primary analysis. ${ }^{4}$ However, the magnitude of the improvements seen in this analysis of single specific outcome measures is of note; these improvements show that by aiming for guidelinedefined control using either ICS alone or with a combination of ICS and long-acting $\beta_{2}$-agonist (LABA), clinically meaningful improvements are achieved in a range of asthma outcomes. Greater improvements were seen with salmeterol/fluticasone propionate versus fluticasone propionate alone and this finding is consistent with those of previous studies. ${ }^{6.8}$ The differences seen between the treatment arms were clinically significant and the improvements seen in percentage of symptom-free days are of particular note. Overall, combination therapy provided an additional 66 symptom-free days per year when compared with fluticasone propionate alone, and in all strata, symptom-free days and rescue-free days were more probable in patients receiving combination therapy than in patients receiving fluticasone propionate alone.

It has been suggested previously that the minimum improvement in PEF perceptible to the patient is $19 \mathrm{l} / \mathrm{min}$ in PEF. ${ }^{9}$ Considering this value, we can be reasonably confident that the changes in PEF observed in the GOAL study also have a meaningful impact on patients' perception of their disease. The improvements seen in severe exacerbation rates were substantial, resulting in a very low annualised rate. Overall, the improvements seen in both groups were accompanied by improvements in quality of life as measured by the Asthma Quality of Life Questionnaire (AQLQ). AQLQ scores improved throughout the study, and the overall score for all groups and strata approached the value of 6 , suggesting that asthma no longer had a significant impact on quality of life. ${ }^{4,10}$ Further analysis has revealed statistically significant differences between AQLQ scores in Totally Controlled, Well Controlled and Not Well Controlled patients, suggesting that the differences in these control definitions are perceptible to patients. $^{10}$

The differences between treatments were more pronounced in Strata 2 and 3 compared with Stratum 1. This may be due to patients in Strata 2 and 3 experiencing nearmaximal improvements from the ICS doses used prior to screening. In these patients, therefore, it might be expected that the addition of an LABA would have a comparatively greater effect.

The magnitude of improvement in many outcomes was greater over Weeks 1-52 compared with Weeks 1-12, which suggests increased benefits from sustaining treatment (following a period of stepping-up the dose), and may also suggest that the benefits are dose-dependent. One possibility is that these benefits may result from the effects of long-term treatment with ICS on airway inflammation and remodelling. Although not studied in the GOAL study, a number of studies have shown that long-term treatment with ICS leads to a reduction in airway inflammation and hyper-responsiveness, and a reversal in airway remodelling. ${ }^{11-13}$ In the study by Ward et al., although a reduction in inflammatory cell counts was seen after three months of treatment with fluticasone propionate, improvements in airway remodelling were not seen until 12 months of treatment." The effects of ICS therapy in airway remodelling are a source of controversy and this issue requires further research.

The GOAL study used a stable dosing treatment strategy, following an initial titration period. Further support for a longer-term stable dosing approach has been suggested by the CONCEPT study, in which patients who stepped-up their treatment and were stable initially on salmeterol/fluticasone propionate (50/250 mcg twice daily) had substantially better long-term outcomes than patients randomised to adjusted dosing with formoterol/budesonide (6/200 mcg), which allowed patients to reduce their treatment to once daily. ${ }^{14}$

One finding of particular note from these additional analyses was that the median percentage of rescue medication-free days was consistently higher than the median percentage of symptom-free days. It has previously been reported that patients may 'adapt' to persistent symptoms and may therefore consider themselves to be 'Well Controlled' despite experiencing daily symptoms. ${ }^{2}$ This suggests that patients may not use rescue medication when it would be beneficial but, rather, may endure the symptoms to which they have become accustomed. In the GOAL study, asthma symptoms were specifically quantified each day, and therefore patients could be expected to give a more truthful estimation of symptoms. The recording of rescue use relied more on the patient's own perception of symptoms, and therefore the percentage of rescue-free days appears lower. Furthermore, it 
is possible that some patients may be more likely to use rescue medication for certain symptoms than others, due to their perceived responsiveness of different symptoms to rescue SABA use.

The present analysis has a number of limitations. First, some analyses reported here were post hoc analyses of prospectively collected data. However, most analyses were a priori. In addition, individual measures do not provide a complete picture of asthma control. Accordingly, the results of this analysis do not suggest that asthma should be assessed using individual measures only, but that these measures complement and reinforce the previous findings. This study did not include the opportunity to step down therapy. Therefore, it remains to be determined whether treatment could be stepped down and, if so, how this should be done to maintain asthma control once achieved.

In conclusion, international surveys such as the AlR surveys have demonstrated that good control of asthma is rare. This additional analysis of the GOAL dataset shows that, in a patient population with a wide range of disease severity, aiming for guideline-defined control using either ICS alone or $I C S+L A B A$ resulted in clinically meaningful improvements in a range of asthma outcomes. Patients on salmeterol/fluticasone propionate benefited substantially more than those receiving fluticasone propionate alone. These data support the recent recommendation in international guidelines that patients are assessed according to the level of control and are then treated with stable dosing, following stepping-up of treatment to achieve and then maintain asthma control. ${ }^{15}$ These results should act as a spur to future efforts in clinical practice to reduce residual morbidity in persistent asthma.

\section{Funding}

The GOAL study was funded by GlaxoSmithKline

\section{Conflict of interest declaration}

AAW has received education, research and travel grants, and remuneration for lectures, consultancy and advisory boards, from GlaxoSmithKline (GSK). He has also received remuneration for these activities from Chiesi Pharmaceuticals, Novartis, Oriel Therapeutics, and Schering-Plough.

$A B-$ no conflicts of interest declared

WB - no conflicts of interest declared

MRG is an employee of GSK. He co-ordinated the study and assisted with the reporting of the study results.

JB received honoraria for his involvement in the GOAL study

EDB has received remuneration from GSK for lectures, membership of advisory boards and consultancy. He has also received remuneration for these activities from AstraZeneca, Hoffman-la-Roche, ALTANA, Boehringer Ingelheim, and Pfizer.

\section{References}

1. Global Initiative for Asthma (GINA). Global Initiative for Asthma (GINA) Global Strategy for Asthma Management and Prevention. 2005.

2. Rabe KF, Adachi M, Lai CK, et al. Worldwide severity and control of asthma in children and adults: the global asthma insights and reality surveys. J Allergy Clin Immunol 2004;114(1):40-7.

3. National Asthma Education and Prevention Program. Expert Panel Report: Guidelines for the diagnosis and management of asthma: Update on selected topics. 2002.

4. Bateman ED, Boushey HA, Bousquet J, et al. Can guideline-defined asthma control be achieved? The Gaining Optimal Asthma controL study. Am J Respir Crit Care Med 2004;170(8):836-44.

5. Van Elteren PH. On the combination of independent two sample tests of Wilcoxon. Bulletin of the International Statistical Institute 1960;37:353-61.

6. Busse W, Koenig SM, Oppenheimer J, et al. Steroid-sparing effects of fluticasone propionate 100 microg and salmeterol 50 microg administered twice daily in a single product in patients previously controlled with fluticasone propionate 250 microg administered twice daily. J Allergy Clin Immunol 2003;111(1):57-65.

7. Jenkins C, Woolcock AJ, Saarelainen $P$, Lundback B, James $M H$. Salmeterol/fluticasone propionate combination therapy 50/250 microg twice daily is more effective than budesonide 800 microg twice daily in treating moderate to severe asthma. Respir Med 2000;94(7):715-23.

8. Kavuru M, Melamed J, Gross G, et al. Salmeterol and fluticasone propionate combined in a new powder inhalation device for the treatment of asthma: a randomized, double-blind, placebo-controlled trial. J Allergy Clin Immunol 2000;105(6 Pt 1):1108-16

9. Santanello NC, Zhang J, Seidenberg B, Reiss TF, Barber BL. What are minimal important changes for asthma measures in a clinical trial? Eur Respir $J$ 1999;14(1):23-7.

10. Bateman ED, Bousquet J, Keech ML, Busse WW, Clark TJ, Pedersen SE. The correlation between asthma control and health status: the GOAL study. Eur Respir J 2007;29(1):56-62

11. Ward C, Pais M, Bish R, et al. Airway inflammation, basement membrane thickening and bronchial hyperresponsiveness in asthma. Thorax 2002;57(4):309-16.

12. Reddel HK, Jenkins CR, Marks GB, et al. Optimal asthma control, starting with high doses of inhaled budesonide. Eur Respir J 2000;16(2):226-35.

13. Beckett PA, Howarth PH. Pharmacotherapy and airway remodelling in asthma? Thorax 2003;58(2):163-74.

14. Fitzgerald JM, Boulet LP, Follows R. The CONCEPT Trial: A 1-year, multicenter, randomized, double-blind, double-dummy comparison of a stable dosing regimen of salmeterol/fluticasone propionate with an adjustable maintenance dosing regimen of formoterol/budesonide in adults with persistent asthma. Clin Ther 2005;27(4):1-14

15. Global Initiative for Asthma (GINA). Global Strategy for Asthma Management and Prevention. [Accessed 2007 Jan 09] . 2006. www.ginasthma.org, Global Initiative for Asthma.

\section{Available online at http://www.thepcrj.org}

